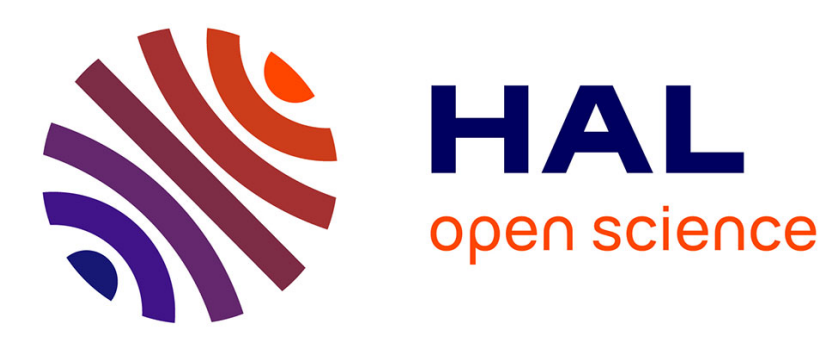

\title{
L'Allemagne et l'après-Yalta ou les hauts et les bas d'un double anniversaire
}

\author{
Anne-Marie Le Gloannec
}

\section{To cite this version:}

Anne-Marie Le Gloannec. L'Allemagne et l'après-Yalta ou les hauts et les bas d'un double anniversaire. Politique étrangère, 1989, 54 (3), pp.411-421. 10.3406/polit.1989.3870 . hal-01009358

\section{HAL Id: hal-01009358 \\ https://hal-sciencespo.archives-ouvertes.fr/hal-01009358}

Submitted on 17 Jun 2014

HAL is a multi-disciplinary open access archive for the deposit and dissemination of scientific research documents, whether they are published or not. The documents may come from teaching and research institutions in France or abroad, or from public or private research centers.
L'archive ouverte pluridisciplinaire HAL, est destinée au dépôt et à la diffusion de documents scientifiques de niveau recherche, publiés ou non, émanant des établissements d'enseignement et de recherche français ou étrangers, des laboratoires publics ou privés. 


\title{
L'Allemagne et l'après-Yalta ou les hauts et les bas d'un double
} anniversaire

In: Politique étrangère $N^{\circ} 3$ - 1989 - 54e année pp. 411-421.

\begin{abstract}
Résumé
La politique des petits pas et des concessions, inaugurée par la coalition social-démocrate et libérale et poursuivie par la coalition conservatrice et libérale à partir de 1982, avait permis de tisser un dense réseau de liens interallemands qui n'avaient pas lâché au cœur de la seconde guerre froide des années 80 . Au lieu de geler les relations interallemandes après que le Bundestag eut accepté de déployer les fusées américaines sur le sol de République fédérale, le gouvernement de Berlin-Est préféra intensifier ses relations avec Bonn et même laisser les Allemands de l'Est se rendre plus facilement à l'Ouest. Etait-ce la conséquence immédiate des concessions ouest-allemandes, le changement induit par le rapprochement qu'Egon Bahr avait souhaité ? Le raidissement actuel démontre les limites de la politique d'alors que des ambiguïtés sous-tendaient : quel changement et quel rapprochement implique la formule d'Egon Bahr? A quel point les Allemagnes doivent-elles se rapprocher et à quel point changer?
\end{abstract}

\section{Abstract}

A Reviuew of Ups and Downs in Post-Yalta Germany on its Double Anniversary, by Anne-Marie Le Gloannec The policy of progress by small steps and concessions inaugurated by the Social Démocratie and Liberal Coalition and folio wed since 1982 by the Conservative/Liberal Coalition created a solid network of ties between the two Germanies which has proved robust. Instead of freezing inter-German relations after the installation of American missiles in the Federal Republic, East Berlin chose to intensify ties with Bonnu and even to allow East Germans easier access to the West. Was this direct consequence of West German concessions, the change induced by closer relations for which Egon Bahr had hoped? The present hardening of attitudes shows the limitations of those policies: how much change and rapprochement has the Egon Bahr formula in fact produced? When will the real change and rapprochement take place between the two Germanies?

Citer ce document / Cite this document :

Le Gloannec. L'Allemagne et l'après-Yalta ou les hauts et les bas d'un double anniversaire. In: Politique étrangère N³ - 1989 54e année pp. 411-421.

doi : 10.3406/polit.1989.3870

http://www.persee.fr/web/revues/home/prescript/article/polit_0032-342X_1989_num_54_3_3870 
Anne-Marie LE GLOANNEC *

\section{L'Allemagne de l'après-Yalta ou les hauts et les bas d'un double anniversaire **}

\section{A}

la veille du $40^{\circ}$ anniversaire de sa fondation, la RDA s'emmure. En 1961 déjà, les dirigeants est-allemands avaient érigé un mur autour de Berlin-Ouest pour enrayer l'hémorragie qui vidait la RDA de ses habitants. Vingt-huit ans plus tard, les mêmes effets appellent la même politique, signe d'impuissance. Erich Honecker, celui-là même à qui Walter Ulbricht avait confié la construction du Mur, ferme les frontières du pays pour mettre fin aux folles échappées de l'été et de l'automne 1989: jusqu'au début du mois d'octobre, plus de 30000 Allemands de l'Est auront trouvé refuge en République fédérale, transitant par la Hongrie, la Tchécoslovaquie et la Pologne; au total, près de 200000 d'entre eux auront, légalement ou non, gagné la RFA en cette année 1989. Pourtant Erich Honecker avait tenté une certaine libéralisation, autorisant dès 1984, voyages et départs. En 1986, plus de 2 millions d'Allemands de l'Est purent faire une incursion en deçà de l'Elbe; en 1987, ils furent 5 millions et presque 7 en $1988^{\circ}$. Cette libéralisation - ou cette prétendue libéralisation - comporte cependant des revers. D'une part, ne se rendent en République fédérale que ceux qui $y$ ont parents ou amis ou qui y vont pour affaires. Ce faisant, le régime instaure deux classes de citoyens tout en prescrivant aux uns et aux autres les règles d'un jeu limité : s'il fait preuve de libéralité, il n'est pas question de liberté. D’autre part, la politique de libéralisation s'accompagne paradoxalement d'un durcissement : à mesure que les Allemands de l'Est élargissent leurs horizons, ils apprennent la démocratie et se veulent citoyens. Depuis la Pentecôte 1987, où de jeunes Berlinois de l'Est avaient manifesté leur colère contre ceux qui les empêchaient d'écouter un concert à l'Ouest, protestations et réclamations n'ont cessé : la RDA entrait alors dans une ère de turbulences auxquelles Erich Honecker n'oppose qu'un raidissement, encourageant par là même départs et protestations, voire la constitution de mouvements d'opposition qui ne cherchent plus désormais le patronage de l'Eglise protestante.

\footnotetext{
* Chercheur au CNRS rattaché au Centre d’études el de recherches internationales (CERI) de la Fondation nationale des sciences politiques, Paris.

* Ce texte est une version adaptée et actualisée de la conclusion de La Nation orpheline. Les Allemagnes en Europe, Calmann-Lévy, Paris, octobre 1989.

1. En fait, il faudrait parler de voyages car ceux-ci sont comptabilisés et non les voyageurs.
} 


\section{La crise est-allemande}

Au durcissement du régime répond un froid interallemand. Des Verts aux conservateurs, la classe politique ouest-allemande n'a pas ménagé ses critiques à l'égard d'une politique qu'elle juge, à juste titre, répressive et sclérosée. Mais elle est divisée sur la conduite à adopter, démontrant ainsi la fragilité du fameux consensus ouest-allemand en matière de Deutschlandpolitik. Ces divisions traversent de surcroît les partis et déchirent avant tout le parti social-démocrate qui voulut poursuivre le plus loin le dialogue avec Berlin-Est, négligeant parfois d'autres forces sociales [1]. Aux conservateurs qui, à la suite d'incidents au Mur en mars 1989, annulèrent des déplacements prévus en RDA, les sociaux-démocrates recommandèrent prudence et modération. Il faudra attendre septembre 1989 - où la crise du régime bat son plein - pour que certains exigent une révision de la politique menée jusqu'alors. Certes le SPD fait valoir que son dialogue avec le SED a pu encourager l'opposition en RDA puisqu'il exhortait celui-ci à s'ouvrir aux critiques et aux réformes. Néanmoins, à droite et à gauche du parti, quelques-uns en dénoncent également les limites : au changement par le rapprochement (Wandel durch Annäherung), conçu par Egon Bahr dès 1963, le député Norbert Gansel veut substituer un changement par la distance (Wandel durch Abstand) ${ }^{2}$. Si tous ne croient pas que leur parti a pêché par complaisance envers le SED, Willy Brandt n'en veut pas moins annoncer l'aube de temps nouveaux. Dans un discours commémorant le $50^{c}$ anniversaire de l'invasion de la Pologne par les troupes de Hitler et de Staline, l'ancien chancelier déclarait récemment : "Une ère touche à son terme, une ère pendant laquelle notre relation avec l'autre Etat allemand consista avant tout à préserver, à maints petits pas, la cohésion des familles divisées et celle de la nation » [2].

Cette politique des petits pas et des concessions, que la coalition socialdémocrate et libérale avait inaugurée et que la coalition conservatrice et libérale endossa après sa venue au pouvoir en 1982, avait permis de tisser un dense réseau de liens interallemands qui n'avaient pas lâché au cour de la seconde guerre froide des années 80. Au lieu de geler les relations interallemandes après que le Bundestag eut accepté de déployer les fusées américaines sur le sol de République fédérale, le gouvernement de BerlinEst préféra intensifier ses relations avec Bonn et même laisser les Allemands de l'Est se rendre plus facilement à l'Ouest. Etait-ce la conséquence immédiate des concessions ouest-allemandes, le changement induit par le rapprochement qu'Egon Bahr avait souhaité ? Le raidissement actuel démontre les limites de la politique d'alors - social-démocrate, libérale et conservatrice - que des ambiguïtés sous-tendaient : quel changement et quel rapprochement implique la formule d'Egon Bahr? A quel point les Allemagnes doivent-elles se rapprocher et à quel point changer? D'une part, lequel des deux termes est le principal, le changement ou le rapprochement, la réunification des Allemagnes ou la libération de la RDA ? D'autre part, rapprochement et changement peuvent-ils s'arrêter en deçà d'une réunification ou d'une libéralisation et encore peut-il y avoir libération sans réunification?

2. Sans parler du « rapprochement par copinage " (Wandel durch Anbiederung), formule qu'a forgée le conservateur Volker Rühe pour critiquer la politique du SPD. 
Si les élites ouest-allemandes s'inquiètent de l'avenir est-allemand et des relations interallemandes, elles ont parfois paru hésiter entre changement et rapprochement : ce n'est pas là l'un des moindres paradoxes de la politique de Bonn. Le changement du régime est-allemand et le rapprochement des deux Allemagnes requièrent la stabilité des relations interallemandes et celle du gouvernement est-allemand. Dans cette perspective, subventions et concessions multiples doivent servir à consolider l'Etat est-allemand. Paradoxalement toutefois, elles déstabilisent la RDA autant qu'elles la stabilisent : plus la population s'enrichit, plus elle multiplie les revendications. Après le pain, la liberté... Mais, justement, le gouvernement est-allemand peut mettre en avant sa propre instabilité pour exiger davantage de Bonn ${ }^{3}$. Les pièges de la stabilisation sont bien réels : les élites politiques ouest-allemandes risquent non seulement de multiplier les concessions à vide ou de surestimer l'indépendance de Berlin-Est par rapport à Moscou, mais encore de préférer la stabilité présente aux changements futurs, de sacrifier en somme les modifications actuelles aux transformations possibles et de s'adresser trop souvent aux gouvernants plutôt qu'aux gouvernés. En 1984, après que des Allemands de l'Est avaient trouvé refuge à la représentation permanente de la République fédérale à Berlin-Est et dans certaines ambassades en Europe de l'Est, Bonn décida, de concert avec les autorités estallemandes, d'en limiter l'accès pour éviter de nouveaux incidents. En juillet 1989, il ferma une fois de plus représentation et ambassades avant de constater qu'il ne pouvait repousser le flux des réfugiés. Certaines démarches ou attitudes sont cependant inutiles: pendant longtemps, une partie des Verts notamment et les sociaux-démocrates plus que les libéraux et les conservateurs ne préférèrent-ils pas éviter l'opposition est-allemande ${ }^{4}$ ? Mais il n'en demeure pas moins que, confrontés à la crise aiguë du pouvoir est-allemand, les dirigeants ouest-allemands ne peuvent vouloir autre chose que calmer les esprits et maintenir ouvertes les voies d'un dialogue avec Berlin-Est.

La crise du régime est-allemand menace en effet d'ébranler les fondations centre-européennes: alors même que l'absence de réformes déstabilise le régime et conduit ceux qui n'y croient plus à se réfugier en République fédérale, une libéralisation du régime est-elle encore possible et toute réforme ne pose-t-elle pas la question de l'unité allemande à l'heure où les attentes s'exacerbent? L'un des idéologues du SED, Otto Reinhold, déclarait récemment que, privée d'un régime socialiste, la RDA perdrait sa raison d'être. Tout retrait de l'Etat laisserait le champ libre à une société qui, entre espace privé et emprise de l'Etat, n'a guère pu se définir et commence à peine à s'organiser autour de mouvements d'opposition : un élan national risquerait de l'embraser et de l'emporter vers les seules structures qu'elle connaisse et que souvent elle désire, celles de la République fédérale. Selon un récent sondage, $64 \%$ des Allemands de l'Est - et $88 \%$ des Allemands de l'Ouest - souhaitent la réunification, les plus âgés, ceux qui ont encore une mémoire d'Allemagne, plus que les jeunes [3]. Mais ce sont ceux-ci qui osent fuir en République fédérale où ils auront

3. Ainsi maintenant la reconnaissance pleine et entière de la citoyenneté est-allemande afin de couper l'herbe sous le pied de ces Allemands de l'Est qui veulent gagner la République fédérale.

4. Une opposition non structurée en mouvements, pour ne pas dire partis, jusqu'à cet automne. 
parfois quelques difficultés à s'adapter mais dont ils connaissent déjà la langue et aussi, bien qu'imparfaitement par la télévision, les mœurs et les codes.

Cependant le nombre d'Allemands de l'Ouest qui souhaitent la réunification ne cessent d'augmenter même si des réactions négatives se font jour à l'encontre de ceux qui « nous prennent notre travail »: mais, à l'heure où les réfugiés venus de RDA promettent un boom économique et démographique, on semble mieux s'accommoder des Allemands de l'Est que de ceux issus de Pologne, de Roumanie ou d'Union soviétique, aux origines et à la culture plus lointaines. Toujours est-il que lorsqu'au premier trimestre 1984, près de 40000 Allemands de l'Est furent autorisés à émigrer en République fédérale, seul un cinquième des Allemands de l'Ouest avaient réagi avec sympathie. En septembre 1989 cependant, la chaleur de l'accueil s'est faite plus vive - comme les émotions. Si l'on peut souhaiter la réunification comme on souhaite qu'il fasse beau, la question allemande n'en est pas moins posée, à la fois par la crise du régime - et rappelons-le, dès la première moitié des années 80 parce que les Allemands s'étaient rapprochés alors que l'Est et l'Ouest engageaient une seconde guerre froide - et aussi parce que, depuis la venue de Gorbatchev au pouvoir et surtout depuis la Table ronde polonaise et la sortie de Janos Kadar, le Vieux Continent se craquèle.

Certes les atteintes portées à l'ordre de l'après-guerre ne sont pas nouvelles : si les révolutions avortèrent à l'Est et si les grandes conférences de l'après-guerre conduisirent finalement à la reconnaissance du statu quo, il n'en demeure pas moins que, dès les années 60, la France à l'Ouest et la Roumanie à l'Est voulurent faire éclater les blocs. Plus tard, dans les années 70 , les partis eurocommunistes entendirent refaire l'unité du continent en ressoudant le mouvement ouvrier. Cependant les anciens courants resurgissaient et les liens gémellaires se renouaient entre Vienne et Budapest... comme entre les Allemagnes.

\section{Question allemande et question européenne : unité ou division, puissance et rôle}

A l'aube du troisième millénaire toutefois, il ne s'agit plus seulement de l'Europe centrale et les atteintes portées à l'ordre de Yalta ${ }^{5}$ ne sont plus seulement culturelles et politiques. L'affaiblissement de l'emprise soviétique en Europe de l'Est et l'occidentalisation de la Pologne et de la Hongrie, voire leur association possible à la Communauté économique européenne, la restructuration plus ou moins subtile, plus ou moins violente de l'Alliance atlantique dont les querelles portant sur le déploiement des FNI ou sur la modernisation des Lance ne sont que des prodromes, la constitution de l'Europe de 1993, l'ouverture enfin de grandes négociations militaires annoncent des bouleversements européens. La RDA persistera-t-elle à choisir l'isolement alors que des réformes économico-politiques s'imposent si elle entend conserver les avantages que lui confère son attachement encore privilégié au Marché commun? Toutes choses étant égales par ailleurs,

5. Bien que l'Europe n'ait pas été divisée à Yalta, il s'agit d'une expression commode. 
pourra-t-elle se maintenir à l'écart du courant général ${ }^{6}$ ? Et bien qu'Egon Krenz entende maintenir le monopole du SED, des réformateurs ou tout simplement la rue ne veulent-ils pas aller beaucoup plus loin?

Quelle que soit l'issue de bouleversements qui recréeront ou non l'unité du continent européen, celle-ci se dessine dans les faits - décrits plus haut et dans les dires - du «sortir de Yalta » de François Mitterrand à la «maison commune » de Mikhaïl Gorbatchev. Cette fluidité nouvelle de l'ordre européen renvoie d'ores et déjà à l'ordre allemand: l'unité européenne se fera-t-elle — si elle se fait — avec celle de l'Allemagne ? L'Europe désormais pose la question allemande. Les élites ouest-allemandes ne l'ignorent pas qui ont entretenu la question allemande au fil des années, même si la coalition social-démocrate et libérale, au pouvoir pendant treize ans, ne voulut pas revendiquer expressément l'unité allemande. Pourtant, l'ancien chancelier Brandt, qui avait rayé le mot unité de son vocabulaire, ne déclarait-il pas récemment : "Etant donné la fluidité de la situation européenne, étant donné qu'une plus grande coexistence s'instaure entre les différentes parties de l'Europe, ce serait contraire à l'histoire de penser que, justement dans le cas des deux Etats allemands, les choses doivent rester comme elles sont aujourd'hui »[4].

Dès la controverse qui opposa partisans et détracteurs du déploiement en République fédérale de fusées américaines à moyenne portée, certains, à gauche puis à droite de l'échiquier politique, saisirent l'occasion pour lier désarmement et question allemande. La querelle portant sur la modernisation des fusées américaines à courte portée donna à celle-ci un tour plus aigu : fallait-il accepter de moderniser ces engins, alors que le retrait des fusées à moyenne portée, assurant le lien entre théâtre européen et capacités stratégiques américaines, réduisait l'automacité de l'escalade et mettait en doute l'engagement américain à défendre l'Allemagne ? Certains, comme Volker Rühe, Alfred Dregger et d'autres encore, furent prompts à craindre que «plus faible est la portée, plus morts seront les Allemands... » ${ }^{7}$. Et le gouvernement d'Helmut Kohl de céder aux pressions publiques et politiques, celles notamment de son libéral ministre des Affaires étrangères, en demandant à l'Alliance de négocier avec l'URSS la réduction, voire, comme le voudrait Hans-Dietrich Genscher, l'élimination, de ces fusées - le fameux troisième zéro.

Quelle que soit la justification des craintes allemandes [5], l'un des arguments invoqués - «...plus morts seront les Allemands » - ne revenait-il pas à mettre en balance engagements atlantiques et communauté nationale ? Indépendamment donc des ententes ou des mésententes interallemandes, la communauté allemande, pour ainsi dire communauté par défaut, pèse d'ores et déjà sur les décisions atlantiques et il pourrait un jour èn aller de même

6. C'est-à-dire en excluant l'hypothèse de contre-coups en Europe de l'Est ou/ct en URSS, la mise à l'écart de Gorbatchev par exemple, ou encore une remise en ordre à laquelle il devrait procéder: s'il a en effet relégué la doctrine Brejnev dans l'ombre, il a néanmoins parlé des pays « socialistes" dont on peut se demander quel est et quel sera le dénominateur commun. Enfin, on peut imaginer la venue au pouvoir en Europe de l'Est de gouvernements nationauxconservateurs bien que l'évolution actuelle n’aille pas en ce sens.

7. "Je kürzer die Reichweite, desto toter die Deutschen... ", formule dont Volker Rühe est le père. 
au sein de la Communauté européenne : une fraction conservatrice voudrait notamment s'assurer que la constitution éventuelle d'une Europe supranationale n'entrave pas la réunification en ajoutant, par exemple, un préalable à tout texte fondant l'unité européenne.

D'une façon générale, les atteintes portées à l'ordre de Yalta, et les espoirs qu'elles suscitent n'ont pas pour unique conséquence de mettre en question la division de l'Allemagne et la nature des liens interallemands. La question allemande n'est pas seulement celle de l'unité ou de la division ; c'est aussi celle de la place et du rôle de l'Allemagne - ou des Allemagnes - en Europe : celle-ci englobe celle-là. Dans la mesure où les élites politiques ouest-allemandes mettent en avant les intérêts de la communauté nationale pour tenter d'imposer à l'OTAN l'ouverture rapide de négociations ou pour obtenir peut-être un troisième zéro, les liens interallemands deviennent objectivement instrument d'influence et le rôle de l'Allemagne l'ultime enjeu. Même si la controverse portant sur la modernisation des Lance opposait les possesseurs d'armes nucléaires, les Etats-Unis, la GrandeBretagne et, marginalement, la France, aux non-nucléaires, ce fut en fait une affaire américano-allemande, match entre les deux Grands de l'Alliance. Elle se conclut, temporairement du moins, non le 30 mai 1989, jour où le sommet de l'OTAN adopta un compromis, mais bien le lendemain lorsqu'à Mayence, le président Bush parla d'un directoire germano-américain.

Plus que toute autre en effet, cette crise américano-allemande modifiait sensiblement le rapport de forces entre les Etats-Unis et la République fédérale. En refusant de moderniser les Lance, voire en cherchant leur élimination, les élites ouest-allemandes rejettent pour la première fois à l'unanimité un certain mode de protection américaine : de source de sécurité, les Etats-Unis deviennent source d'insécurité alors même que le besoin de protection vis-à-vis de l'Est ne se fait plus sentir. La protection américaine s'avère, en ce cas précis, dangereuse et inutile alors que, d'une façon générale, son prix est la dépendance ${ }^{8}$. En refusant de moderniser les Lance, les élites ouest-allemandes s'émancipent quelque peu de la tutelle américaine : l'autonomie est à la fois instrument et objectif de leur politique.

On pourrait en conclure, par boutade, que la République fédérale est devenue une puissance nucléaire a contrario, par l'effet du veto, ou, plus justement, souligner que, dans l'Europe qui se décide - et se dessine - à Vienne notamment, et où les armes auront peut-être une moindre importance que par le passé, l'Allemagne de l'Ouest, puissance civile, aura un poids accru. Toujours est-il qu'en rehaussant le statut de la RFA, le 31 mai 1989, à Mayence, George Bush ne faisait que prendre acte. Mikhaïl Gorbatchev l'imita peu après, lorsqu'à Bonn il évoqua le poids de l'Allemagne dans l'Alliance - pour se féliciter en quelque sorte des résultats du match

8. Pour le public allemand, le double accident de Ramstein et de Remscheid (dans le premier cas, une escadrille italienne s'écrasa lors d'un salon aérien et, dans le second, un avion volant à basse altitude tomba en flammes sur un viliage) symbolise le coût de la dépendance : on accuse un peu vite les avions volant à basse altitude pour les besoins de l'OTAN... Néanmoins, on remarquera que les autres opinions publiques européennes rejettent de la même façon le coût de la dépendance. Le cas allemand est cependant plus aigu puisque s'y mêlent la question de l'intégration quasi totale à l'OTAN et celle de la partition. 
américano-allemand, plutôt favorables à Moscou. Quel beau cadeau les deux superpuissances n'offraient-elles pas à la République fédérale pour ses quarante ans d'existence! Le président de la République, Richard von Weizsäcker, n'avait pas souhaité autre chose lorsque, dans son discours d'anniversaire, il avait affirmé : "nous ne sommes pas (...) le jouet des autres (...) Notre poids politique se définit par l'importance de notre population, par nos aptitudes (Leistungsfähigkeit) et notre stabilité »[7].

\section{Intérêts particuliers et rôle européen}

Mais «nous ne sommes pas une grande puissance", crut-il bon d'ajouter tant il est vrai que la République fédérale, géant économique et puissance moyenne, manifeste des signes de faiblesse, non celles du nain politique qu'elle fut un moment dans l'après-guerre mais celle d'un pays de plus en plus difficile à gouverner. L'affaissement conjoncturel - celui du pouvoir d'Helmut Kohl - masque un mal plus profond et plus durable. La nécessité pour tout gouvernement de reposer sur une coalition, l'émiettement des partis, avec l'apparition des Verts puis celle des Republikaner notamment, l'influence de l'opinion publique que les Eglises et surtout les partis relaient politiquement, la puissance des Länder, à Bonn, à Francfort ${ }^{9}$ ou à Bruxelles, celle du Tribunal constitutionnel, plus impressionnante que celle d'organismes analogues en France : tout concourt à restreindre l'autorité de l'Etat et à alourdir le jeu politique. Certaines limites existaient bien entendu, dans les textes comme dans les faits, depuis la Constitution de la II ${ }^{\mathrm{C}}$ République. Il n'en fallut pas moins attendre la fin des années 60 et les premières initiatives de citoyens pour que les Allemands de République fédérale veuillent investir l'Etat et s'approprier l'Allemagne, redécouvrir leur environnement passé et présent et faire de la Démocratie de Bonn leur démocratie [8]. Il fallut enfin les années 80 pour que la création de nouveaux partis, rivalisant avec les anciens auprès de l'opinion publique, rehausse l'importance de celle-ci dans la vie politique.

L'exercice cependant n'est pas sans risque car autant l'emprise de l'Etat peut s'en trouver justement circonscrite et la démocratie fortifiée, autant l'affaiblissement de l'actuelle coalition, et même de toute coalition au pouvoir, peut nourrir la démagogie. C'est ainsi que, pour parer à la menace des Republikaner qui entament l'aile conservatrice des chrétiens-sociaux et des chrétiens-démocrates, le chancelier Kohl prête aux électeurs de droite une attention qui le paralyse. Alors qu'il aurait dû se rendre en Pologne en juillet 1989 et qu'à sa suite le président de la République aurait marqué le $50^{\mathrm{c}}$ anniversaire de l'invasion du pays par les troupes allemandes et soviétiques, le Chancelier aura, jusqu'au dernier moment, tergiversé, hésitant un moment à accorder un prêt à la Pologne : celui-ci aurait peut-être rebuté certains électeurs dont on a vu, depuis quelques années, qu'ils aimeraient fermer leurs frontières aux immigrants et même à leurs « frères » allemands, de RDA ou d'Europe de l'Est. Certains conservateurs n'ont même cessé de revendiquer la reconstitution de l'Allemagne dans les frontières de 1937 . En 1985 , de concert avec le Chancelier, Volker Rühe rappela qu'en reconnaissant les frontières existantes, la République fédérale avait engagé l'Allemagne future, engagement non juridique mais bien politique [9]. Durant cet

9. Au scin de la Banque fédérale (Bundesbank). 
été de négociations germano-polonaises, Theo Waigel, le successeur de Franz-Josef Strauss et ministre des Finances, revint néanmoins à la charge - au grand dam des Polonais.

Les embarras du gouvernement conduisent cependant les conservateurs éclairés à européaniser une Ostpolitik qu'ils ne croient pouvoir justifier aux yeux de leurs électeurs. Pour ne pas s'enliser plus avant, la présente coalition cherche pour ainsi dire à légitimer sa politique polonaise en l'ancrant dans des cadres occidentaux, formels ou informels, ceux de la Communauté européenne ou de la coopération franco-allemande, ou encore de cette aide conjointe que le président Bush proposa au sommet de Paris. Aussi bien, la majorité des partisans de l'unité allemande veut acquérir l'appui de Bruxelles et de l'Europe alors qu'une minorité seulement voudrait s'assurer la possibilité de quitter la Communauté pour préserver une hypothétique unité. Ainsi, la double appartenance à la Communauté européenne et à l'Alliance atlantique remplit, à côté d'autres fonctions ${ }^{10}$, celle de compenser les faiblesses de l'actuelle coalition ou, plus généralement, de promouvoir les intérêts allemands.

Qu'un pays veuille défendre ses intérêts, au sein de l'Alliance et de la Communauté, quoi de plus naturel ? Et que la République fédérale ne puisse imiter la France de de Gaulle parce qu'en se retirant, elle anéantirait l'Alliance ou la Communauté, quoi de plus connu ? C'est précisément parce qu'elle est une pièce centrale de ces dispositifs qu'elle ne peut faire cavalier seule, mais c'est aussi pour cela qu'elle peut mieux avancer ses pions. Il n'en demeure pas moins qu'une conjonction nouvelle se dessine où confluent la montée en puissance de la République fédérale et les aspirations publiques et politiques à lui voir jouer un rôle adéquat, à l'Est et à l'Ouest, l'ardeur des élites à défendre des intérêts particuliers et encore l'incapacité du gouvernement d'imposer des décisions communautaires et atlantiques à une opinion publique qui les rejette. Hans-Dietrich Genscher la résuma récemment par ces deux mots: Sondergewicht et Sonderinteressen, poids particulier et intérêts particuliers. La République fédérale peut-elle toutefois défendre des intérêts particuliers en prétendant à un rôle européen et international ? Certes, tout gouvernement se livre plus ou moins à cet exercice : la France des années 60 avait brusqué ses partenaires européens et atlantiques, enrayant l'évolution du Marché commun et gênant l'organisation militaire intégrée ; celle des années 80 s'attache à construire l'Europe pour maintenir son rang.

En suspendant le prélèvement à la source sur les revenus de l'épargne, en revenant sur sa décision d'allonger la durée du service militaire, en différant la modernisation des Lance puis en pressant l'ouverture de négociations, toutes mesures que forcèrent l'humeur publique et la fragilité gouvernementale ou la prise en compte des intérêts de la communauté nationale, le gouvernement fédéral revient sur des décisions acquises, formellement ou tacitement, compromet l'Europe fiscale et ébranle la doctrine de la réponse flexible, déjà mise à mal par le retrait des FNI. Alors que l'opinion allemande, certes désireuse de rester dans l'Alliance, perçoit désormais les armes nucléaires non comme un bouclier mais bien comme une menace,

10. Voir infra. 
symbole de la dépendance et de la partition, alors qu'elle s'enthousiasme moins pour l'Europe que pour le réformateur soviétique qui lui promet ce qu'elle attend : la destruction des armes nucléaires - puisque Gorbatchev décrie la dissuasion - et la fin de la division - puisqu'il souhaite la constitution d'une maison commune - alors enfin que l'opposition rêve d'une Europe plus ou moins réunifiée et plus ou moins délivrée d'armes atomiques, on peut se demander comment, à l'avenir, la République fédérale s'acquittera de ses engagements envers l'Alliance et la Communauté. La question est légitime et ne se confond pas avec la crainte d'un nouveau Rapallo dont Richard von Weizsäcker se défendait violemment dans son discours célébrant les quarante ans de la République.

\section{Responsabilités allemandes et engagements européens}

Si la République fédérale veut éviter de "germaniser " l'Alliance et la Communauté en imposant à celles-ci ses intérêts ou ses faiblesses [10], il lui faudra abandonner un peu plus de souveraineté ou d'autonomie, dans le cadre de la Communauté essentiellement, afin de ne pas trop déséquilibrer l'Europe économique et politique. En l'absence d'union économique et monétaire, les monnaies s'alignent sur le mark au sein du SME et les membres de la Communauté suivent la politique de Bonn et de Francfort, pour le pire et pour le meilleur. En 1988, le ministre italien du Trésor, Giulano Amato, n'avait pas craint de comparer l'Europe des années 90 à une nouvelle Grande Allemagne [11]. Une union économique et monétaire, où les autorités ouest-allemandes composeraient avec leurs partenaires, encadrerait au contraire la montée en puissance de la République fédérale, aplanissant pour ainsi dire les ressentiments étrangers et, au bout du compte, les exaspérations ouest-allemandes à l'égard de ceux-ci. De même, un ancrage plus profond dans la Communauté européenne préviendrait à la fois un Sonderweg allemand et désamorcerait les craintes alliées d'un nouveau Rapallo. C'est pour ces raisons politiques précisément que le chancelier Kohl et son ministre des Affaires étrangères se sont tant engagés à Bruxelles, lors de la dernière présidence allemande; c'est pour cela aussi qu'ils pressent la création d'une Banque centrale européenne, contraire, pour Karl-Otto Pöhl, à la raison économique.

N'est-ce pas toutefois paradoxal que d'encourager la République fédérale à abandonner certains droits souverains alors même qu'élites et opinion allemandes souhaitent voir le pays jouer un plus grand rôle ? Les alliés de la République fédérale devront prendre en compte ces aspirations à plus de souveraineté, même si celles-ci - ou bien plutôt parce que celles-ci trahissent parfois des ressentiments : à l'impatience que les contraintes otaniennes suscitent, symbolisées en quelque sorte par le double accident de Ramstein et de Remscheid, à la confusion entre «alliance » et "occupation », les alliés de la République fédérale ne peuvent apporter que des remèdes fragmentaires, preuves de bonne volonté. La véritable réponse se trouve ailleurs. Elle consiste à accepter non seulement que la République fédérale joue un rôle à sa mesure - comme le préconise le président Bush - mais encore qu'elle puisse se déterminer librement.

La question se pose désormais, a-t-on dit, parce que le Vieux Continent recouvre une fluidité perdue depuis la guerre. Certains Etats, plus que d'autres, ont un intérêt immédiat à transformer le statu quo: l'Allemagne 
plus que la France, l'Europe de l'Est plus que l'Europe de l'Ouest, l'Union soviétique plus que les Etats-Unis - l'Allemagne pour retrouver l'unité ou, du moins, pour obtenir que les Allemands de l'Est puissent décider de joindre ou non leur sort à celui des Allemands de l'Ouest; l'Europe de l'Est pour se libéraliser et se libérer de l'emprise soviétique; l'Union soviétique enfin pour se constituer non un empire européen de Brest à Brest mais une sorte d'avant-cour, facile et prospère. A long terme, les objectifs divergent ; à court terme, certaines tactiques convergent. En déclarant, dans le communiqué commun signé à Bonn en juin 1989, qu'ils travailleraient à surmonter la coupure de l'Europe, Mikhaïl Gorbatchev et Helmut Kohl ont voulu souligner cette convergence, assimilant ordre de paix européen (europäische Friedensordnung) et maison commune européenne (gemeinsames europäisches Haus) ${ }^{11}$.

En se proposant de bâtir une maison commune, Mikhaïl Gorbatchev ne doit pas ignorer qu'il entretient la question allemande. Les petites phrases russes n'auront pas manqué [12] même si, lors de la visite du chancelier Kohl à Moscou en octobre 1988 comme lors de ses propres visites à Bonn et à Berlin-Est, Gorbatchev se sera félicité de l'existence de l'autre Etat allemand. Au cours de la présente crise d'ailleurs, Moscou aura pris le parti de Berlin-Est - tout en suggérant la nécessité de réformes. Il se peut néanmoins que les Soviétiques se ravisent : ils n'ont, après tout, guère à craindre d'une Allemagne réunifiée, puissance civile désireuse d'aider aux transformations économiques notamment. Il se peut aussi que les Allemands d'entre Elbe et Oder posent bruyamment ou encore violemment la question allemande [13]. Il nous faudra alors tenir nos promesses historiques pour ne pas nous aliéner l'Allemagne: les Allemands devront exercer leur droit à l'autodétermination, se confédérer ou peut-être se réunifier. La réunification avivera les tensions, elle mettra en question l'ordre européen, les blocs, militaires plus qu'économiques, et les configurations politiques : on s'interrogera sur le rôle des Etats-Unis, sur celui de l'Union soviétique, de l'Allemagne ou des Allemagnes, ou encore sur le poids des armes et sur la nécessité de la dissuasion. Allemands et alliés devront se mettre d'accord sur les réponses.

Si, en République fédérale, l'opinion publique dit vouloir l'unité, si elle entend troquer la dissuasion nucléaire pour une hypothétique dissuasion conventionnelle, elle n'en est pas moins attachée à l'Alliance et ses dirigeants connaissent la pleine mesure de leurs responsabilités. A une audience qui l'applaudit quasi unanimement, Erhard Eppler conseilla récemment de choisir, en deçà de l'unité, une formule nouvelle qui ne bouleverse pas l'Europe car "si les Slovènes, les Serbes et les Croates veulent vivre en un seul Etat, cela ne concerne qu'eux... Le mur hideux de fer et de béton qui divisa l'Allemagne en 1945 soutient la maison (européenne) plus que nous le souhaitons. Qui veut l'abattre, doit repenser l'équilibre de la maison et même peut-être la reconstruire. Ce qu'il adviendra de l'Allemagne concerne tous les Européens" [14].

(octobre 1989)

11. La première formule est celle des Allemands de rOuest, la seconde celle des Soviétiques. 


\section{NOTES BIBLIOGRAPHIQUES}

[1] Sur ce point, on se reportera à Anne-Marie Le Gloannec, La Nation orpheline. Les Allemagnes en Europe, Calmann-Lévy, Paris, 1989, partie IV : « Le dialogue entre Bonn et Berlin-Est ».

[2] Cité par Eckhard Fuhr, « Nach einem Irrweg Rufe zur Besinnung. Schmerzliche Erfahrungen der SPD mit ihrer Deutschlandpolitik », Frankfurter Allgemeine Zeitung, 22 septembre 1989.

[3] Voir «Wann kommt die Wiedervereinigung? », Quick, n 40, 28 septembre 1989. Si les Allemands de l'Est sont moins favorables que ceux de l'Est à la réunification, c'est qu'ils en attendent moins de bénéfices personnels (48\% contre $58 \%$ ) soit qu'ils veuillent un socialisme démocratique, soit qu'ils craignent la société de concurrence qui les priverait de leur quiétude.

[4] Willy Brandt, «Westeuropa ist kein Ersatzvaterland ", interview avec la Süddeutsche Zeitung, supplément consacré au $40^{\mathrm{e}}$ anniversaire de la RFA, 24 mai 1989, p. 11.

[5] Ces craintes sont en partie justifiées puisque, on l'a dit plus haut, le retrait des FNI affaiblissait l'automaticité de l'escalade, alors que les FNI et le "paquet d'armes qui les accompagnait devaient précisément assurer la flexibilité de la réponse... flexible. Mais ces craintes sont aussi injustifiées dans la mesure où l'imbrication des troupes américaines, canadiennes et européennes sur le sol allemand ferait de toute guerre une guerre générale. Voir Anne-Marie Le Gloannec, La Nation orpheline, op. cit., partie IV.

[6] Un récent ouvrage publie une foule de sondages, effectués en RFA et en URSS : la perception d'une menace soviétique s'est évanouie en République fédérale. Voir Klaus Liedtke, Der neue Flirt. Russen und Deutsche auf dem Weg zu veränderten Beziehungen, Stern-Buch im Verlag Gruner + Jahr, Hambourg, 1989.

[7] Voir le discours du président de la République lors des cérémonies célébrant le $40^{\circ}$ anniversaire de la République. Texte dans la Süddeutsche Zeitung, 26 mai 1989.

[8] Voir Anne-Marie Le Gloannec, La Nation orpheline, op. cit., partie I.

[9] Voir Anne-Marie Le Gloannec, La Nation orpheline, op. cit., parties II et IV.

[10] C'est Josef Joffe qui parle de «germanisation» dans «Falscher Konflikt zur falschen Zeit ", Süddeutsche Zeitung, 27 avril 1989.

[11] «Grösseres EWS-Brandbreiten für alle. Rom befürchtet ein europäisches Grossdeutschland ", Süddeutsche Zeitung, 7 décembre 1989.

[12] Voir par exemple l'article de Karl Feldmeyer, "Souveränität der Staaten Selbstbestimmung der Völker. Moskau sendet widersprüchliche Signale zur deutschen Frage », Frankfurter Allgemeine Zeitung, 16 juin 1989. Karl Feldmeyer rappelle les petites phrases russes, prononcées depuis quelques mois.

[13] Alfred Grosser n'avait-il d’ailleurs pas interpellé les lecteurs du Monde lorsqu'il s'était demandé ce que nous ferions si un Walesa est-allemand agitait l'Allemagne ? Voir Alfred Grosser, "L'interdit ", Le Monde, 20 janvier 1982.

[14] Voir Erhard Eppler (SPD), «Wir muten Europa das Thema Deutschland zu, weil es uns gibt ». Discours prononcé lors de la commémoration anniversaire du 17 juin, texte dans Frankfurter Rundschau, 20 juin 1989. 